\title{
Regional contributions to left ventricular stroke volume determined by cardiac magnetic resonance imaging in cardiac resynchronization therapy
}

Björn Östenson ${ }^{1}$, Ellen Ostenfeld ${ }^{1}$, Anna Werther-Evaldsson², Anders Roijer ${ }^{2}$, Zoltan Bakos ${ }^{3}$, Mikael Kanski ${ }^{1,4}$, Einar Heiberg ${ }^{1,5}$, Håkan Arheden ${ }^{1}$, Rasmus Borgquist ${ }^{3}$ and Marcus Carlsson ${ }^{1 *}$ (D)

\begin{abstract}
Background: Cardiac resynchronization therapy (CRT) restores ventricular synchrony and induces left ventricular (LV) reverse remodeling in patients with heart failure (HF) and dyssynchrony. However, 30\% of treated patients are non-responders despite all efforts. Cardiac magnetic resonance imaging (CMR) can be used to quantify regional contributions to stroke volume (SV) as potential CRT predictors. The aim of this study was to determine if LV longitudinal $\left(S V_{\text {long\% }}\right)$, lateral $\left(S V_{\text {lat } \%)}\right.$, and septal $\left(S V_{\text {sept } \%}\right)$ contributions to SV differ from healthy controls and investigate if these parameters can predict CRT response.
\end{abstract}

Methods: Sixty-five patients (19 women, $67 \pm 9$ years) with symptomatic HF (LVEF $\leq 35 \%$ ) and broadened QRS ( $\geq 120 \mathrm{~ms}$ ) underwent CMR. SV $\mathrm{long}_{\text {\% }}$ was calculated as the volume encompassed by the atrioventricular plane displacement (AVPD) from end diastole (ED) to end systole (ES) divided by total SV. SV lat\%, $_{\text {, }}$ and SV sept\% $_{\text {were calculated as }}$ the volume encompassed by radial contraction from ED to ES. Twenty age- and sex-matched healthy volunteers were used as controls. The regional measures were compared to outcome response defined as $\geq 15 \%$ decrease in echocardiographic LV end-systolic volume (LVESV) from pre- to 6-months post CRT (delta, $\triangle$ ).

Results: AVPD and $\mathrm{SV}_{\text {long\% }}$ were lower in patients compared to controls $(8.3 \pm 3.2 \mathrm{~mm}$ vs $15.3 \pm 1.6 \mathrm{~mm}, P<0.001$; and $53 \pm 18 \%$ vs $64 \pm 8 \%, P<0.01)$. SV $V_{\text {sept } \%}$ was lower ( $0 \pm 15 \%$ vs $\left.10 \pm 4 \%, P<0.01\right)$ with a higher $S V_{\text {lat } \%}$ in the patient group ( $42 \pm 16 \%$ vs $29 \pm 7 \%, P<0.01$ ). There were no differences between responders and non-responders in neither $\mathrm{SV}_{\text {long\% }}(P=0.87), \mathrm{SV}_{\text {lat\% }}(P=0.09)$, nor SV $\mathrm{sept}_{\mathrm{S}}(P=0.65)$. In patients with septal net motion towards the right ventricle $(n=28) \triangle L V E S V$ was $-18 \pm 22 \%$ and with septal net motion towards the LV $(n=37) \triangle L V E S V$ was $-19 \pm 23 \%$ $(P=0.96)$.

Conclusions: Longitudinal function, expressed as AVPD and longitudinal contribution to SV, is decreased in patients with HF scheduled for CRT. A larger lateral contribution to SV compensates for the abnormal septal systolic net movement. However, LV reverse remodeling could not be predicted by these regional contributors to SV.

Keywords: Cardiac resynchronization therapy, Cardiac magnetic resonance, Predictors

*Correspondence: marcus.carlsson@med.lu.se

${ }^{1}$ Clinical Physiology, Department of Clinical Sciences Lund, Lund

University, Skåne University Hospital, Lund, Sweden

Full list of author information is available at the end of the article

\section{Background}

Cardiac dyssynchrony in chronic heart failure (HF) may be restored by cardiac resynchronization therapy (CRT), which induces coordinated contraction in the regulation or exceeds the permitted use, you will need to obtain permission directly from the copyright holder. To view a copy of this licence, visit http://creativecommons.org/licenses/by/4.0/. The Creative Commons Public Domain Dedication waiver (http://creativeco mmons.org/publicdomain/zero/1.0/) applies to the data made available in this article, unless otherwise stated in a credit line to the data. 
left ventricle [1]. The current indications for CRT from European Society of Cardiology (ESC) are symptomatic HF despite optimal medical treatment, left ventricular ejection fraction (LVEF) $\leq 35 \%$ and broadened QRS complex ( $\geq 130 \mathrm{~ms}$ ), preferably with left bundle branch block (LBBB) [2]. In large randomized controlled trials, CRT has been shown to prolong survival, stall progression, and reduce symptoms of HF [3]. However, approximately $1 / 3$ of patients undergoing CRT treatment are objective non-responders [3]. Consequently, there is a need for reliable preoperative predictive factors of response to CRT. Cardiac magnetic resonance imaging (CMR) is gold standard for cardiac volumes and infarct detection. However, neither randomized CMR trials nor more advanced echocardiographic markers have overall not shown a benefit of using imaging beyond LVEF in the selection of CRT candidates $[4,5]$.

Conflicting results have been presented regarding the ability of multi-modality imaging to guide CRT-lead placement. Sommer et al. showed in a single-center randomized controlled trial that a multi-modality imaging approach improved the CRT effect [6]. However, the randomized clinical trial CRT CLINIC study could not show any benefit of assessing the most suitable segments prior to lead placement by using the combination of twodimensional speckle tracking echocardiography (latest segment activation), cardiac CT (suitable cardiac vein anatomy), and CMR (scar evaluation) [7]. Thus, further studies are needed to identify imaging biomarkers that can help select patients more likely to respond to CRT treatment.

CMR can quantify the stroke volume (SV) generated from LV septal net movement in addition to the longitudinal and lateral contributions to SV $[8,9]$. In healthy subjects, the septum contributes to about $7 \%$ of LVSV [10]. However, this may differ in patients with increased QRS duration, where abnormal septal motion often is present [11]. Thus, septal contribution to LVSV may be a prognostic factor of CRT response.

The longitudinal component of LV stroke volume $\left(\mathrm{SV}_{\text {long\% }}\right)$ is generated by the atrioventricular plane displacement (AVPD). Impaired longitudinal ventricular function has been shown to be associated with adverse events, even in the absence of reduced LVEF [12]. However, the possible prognostic value of longitudinal function for predicting treatment response has not been studied in a CRT population.

While other image predictors for response to CRT have been studied, for example apical rocking [13] and myocardial strain [14] with echocardiography, regional contributions to SV with CMR have not been studied in CRT patients. Regional contributions to SV provide, in contrast to other imaging indicators of dyssynchrony, quantitative measurements of how much each of the longitudinal, lateral, and septal displacements generate SV. These three imaging predictors could potentially be used as reliable imaging predictors of response to CRT before implantation, reducing the number of non-responders. Our hypothesis was that measures of regional contributions to SV could be used to select appropriate patients for CRT.

Therefore, the aims of this study were to (1) determine if longitudinal, lateral, and septal contributions to SV in patients with $\mathrm{HF}$ and eligible for CRT differ from healthy controls, (2) investigate if these parameters predict CRTtreatment outcome defined as reverse remodeling at 6 months after CRT implantation, and (3) investigate if there were differences in regional contribution to SV in patients with ischemic versus non-ischemic etiology.

\section{Methods}

\section{Study population}

This is a sub-study of a randomized clinical trial, which prospectively included patients with indication for CRT between 2011 to 2017 at Skåne University Hospital, Sweden (ClinicalTrials.gov: NCT01426321). Indication for CRT was New York Heart Association (NYHA) class II-IV despite optimal medical therapy, QRS-complex $\geq 120 \mathrm{~ms}$ on standard electrocardiogram (ECG), and LVEF $\leq 35 \%$ [15]. Patients with a life expectancy $<12$ months, myocardial infarction within 3 months of enrolment, moderate-severe valve disease, chronic atrial fibrillation, and severely reduced renal function (estimated glomerular filtration rate $<30 \mathrm{ml} / \mathrm{min}$ ) were excluded from the study. The results of the randomized trial were neutral with respect to response to therapy between the two randomized groups, LVESV increase $\geq 15 \%$ at six months in $56 \%$ of patients in the intervention group versus $55 \%$ in controls, $P=0.96$ [15]. Therefore, patients were pooled in this exploratory sub-study. Of a total of 102 patients in the main study, 28 already had devices in situ, and 4 were excluded due to patient refusal or other contraindication, leading to 70 patients undergoing CMR examination. Five of these patients were excluded owing to poor image quality $(n=3)$ or arrhythmia during examinations $(n=2)$. Hence, 65 patients were analyzed (Fig. 1).

Ischemic heart disease was defined as fulfillment of at least one of the following criteria: (1) medical history of myocardial infarction or revascularization (percutaneous coronary intervention or coronary artery bypass grafting), (2) lumen stenosis $\geq 50 \%$ in left main coronary artery or proximal left anterior descending artery, and (3) lumen stenosis $\geq 75 \%$ in any epicardial vessel. Nonischemic heart disease was defined as patients not fulfilling any of these criteria [16]. 


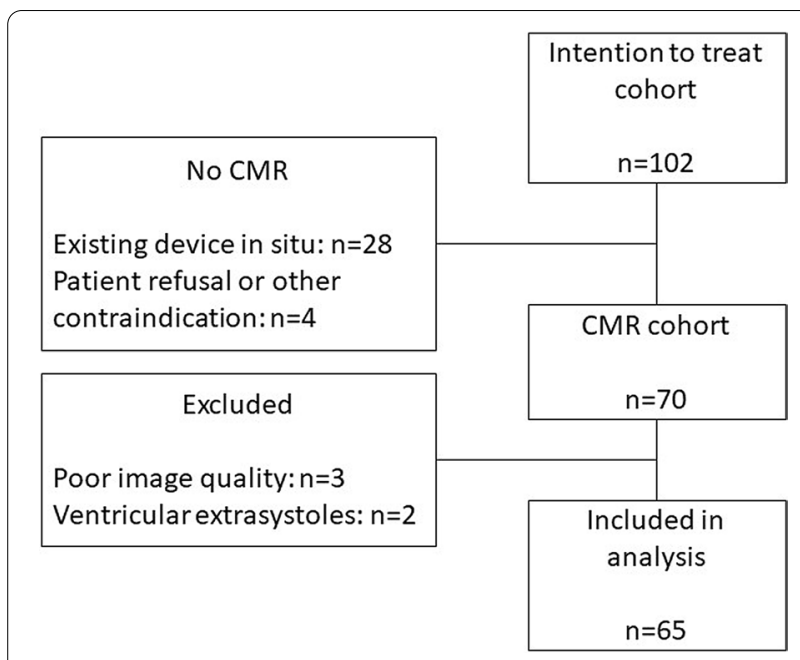

Fig. 1 Flow chart of study participants. CMR cardiac magnetic resonance imaging

As control group, 20 healthy age- and sex-matched individuals ( 8 women, $62 \pm 11$ years) with normal blood pressure $(<140 / 90 \mathrm{mmHg})$, no past cardiovascular diseases, no cardiovascular treatments, and with normal ECG findings were recruited.

\section{Cardiac magnetic resonance imaging}

All subjects underwent CMR examination at baseline using a Philips Intera $1.5 \mathrm{~T}$ or $3.0 \mathrm{~T}$ (Philips Medical System, Best, The Netherlands), or Siemens Aera 1.5 T (Siemens Medical Systems, Erlanger, Germany). Balanced steady-state free precession cine images were collected in the supine position during end-expiratory apnea and included a short-axis cine stack, and long-axis cines in 2-chamber, 3-chamber, and 4-chamber views. CMR was not performed at six months follow-up due to CRT devices were not MRI compatible.

Retrospective ECG triggering was used to acquire images with reconstructed temporal resolution of 30 time frames per cardiac cycle. Typical imaging parameters were TR/TE $3 \mathrm{~ms} / 1.3-1.8 \mathrm{~ms}$, flip angle $45^{\circ}(3 \mathrm{~T})$ or $60^{\circ}(1.5 \mathrm{~T})$, and slice thickness $6-10 \mathrm{~mm}$ with $0-2 \mathrm{~mm}$ slice gap.

In patients, late gadolinium (Gd) enhancement (LGE) images were acquired to quantify the extent of myocardial fibrosis. Intravenous Gd-based contrast of $0.2 \mathrm{mmol} /$ kg (gadoteric acid, Gd-DOTA, Guerbet, Gothia Medical $A B$, Billdal, Sweden) was injected in an antecubital vein after which inversion-recovery or phase sensitive inversion recovery sequences were used to generate LGE images. Typical image parameters were TR/TE 4.2$5.2 \mathrm{~ms} / 1.0-3.3 \mathrm{~ms}$, flip angle $15^{\circ}(1.5 \mathrm{~T})$ or $25^{\circ}(3 \mathrm{~T})$, and slice thickness $6-10 \mathrm{~mm}$ with $0-2 \mathrm{~mm}$ slice gap.

\section{Image analysis}

CMR image analysis was performed using the software Segment v2.0 R5039 (Medviso, Lund, Sweden: http:// segment.heiberg.se) [17]. Short-axis images were delineated according to current consensus document of standardized image interpretation, including trabeculations and papillary muscles in the cavity volumes [18]. LV epicardial and endocardial borders were delineated at end diastole (ED) and end systole (ES) for measurements of ED volume (EDV), ES volume (ESV), and LV mass. SV was defined as EDV-ESV. LVEF was defined as SV/EDV. LV mass was calculated by multiplying the myocardial volume with the muscle density $(1.05 \mathrm{~g} / \mathrm{ml})$. Fibrosis was visually determined as present/absent by an experienced physician ( $>20$ years of experience).

\section{Longitudinal contribution to stroke volume}

The longitudinal contribution to $\mathrm{SV}\left(\mathrm{SV}_{\text {long } \%}\right)$ was measured as previously described $[8,9,19]$. In short, AVPD is defined as the displacement of the atrioventricular plane between ED and ES (Fig. 2). Six standardized landmarks were used to measure the LV atrioventricular plane: from the 4-chamber view: LV inferoseptal and LV anterolateral; from the 3-chamber view: LV anteroseptal and LV inferolateral; and from the 2-chamber view: LV anterior and inferior. The short-axis area comprising the range of the AVPD was calculated using the epicardial delineation in the short-axis views, as previously discussed [9]. The average of the two largest epicardial short-axis areas encompassed by the AVPD motion was multiplied with the AVPD to calculate the absolute longitudinal contribution to SV in $\mathrm{ml}$. The longitudinal contribution relative to total SV $\left(\mathrm{SV}_{\text {long\% }}\right)$ was calculated by dividing the absolute longitudinal contribution to SV (in $\mathrm{ml}$ ) with the total LVSV.

\section{Radial contribution to stroke volume}

The radial contribution to SV was measured as previously described using an in-house developed plugin to the Segment software [20]. In short, the septal contribution to $\mathrm{SV}\left(\mathrm{SV}_{\text {sept } \%}\right)$ was defined by the area enclosed by the septal epicardial contours and the RV insertion points between ED and ES in the short-axis images (Fig. 3). The lateral contribution to $\mathrm{SV}\left(\mathrm{SV}_{\mathrm{lat} \%}\right)$ was defined by the area enclosed by the lateral LV epicardial contours and the RV insertion points in ED and ES in the short-axis images (Fig. 3).

\section{Echocardiography}

Echocardiography was acquired with a Vivid E9 (GE Medical, Horten, Norway) using an M5Sc-D probe with standard projections according to guidelines [21]. 


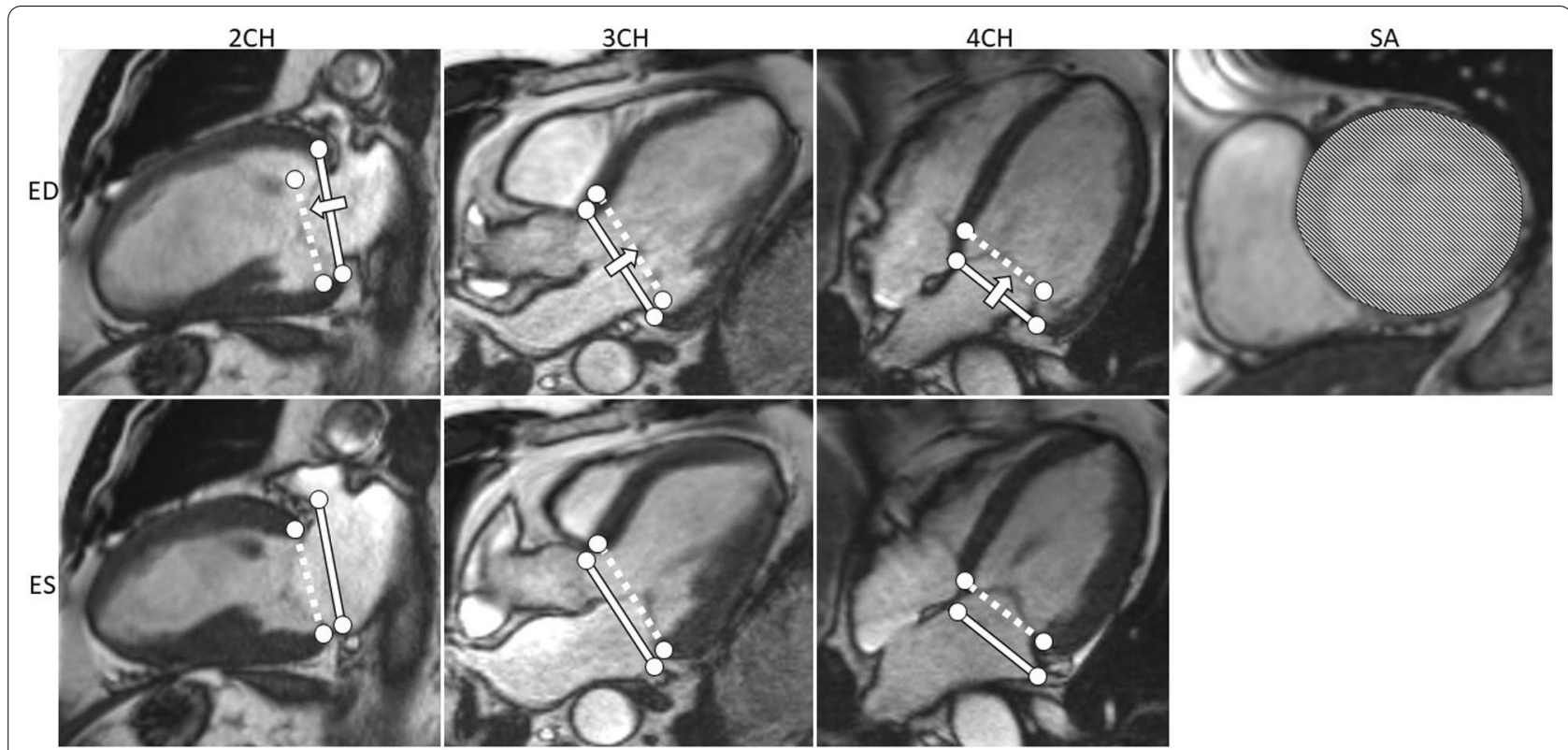

Fig. 2 Atrioventricular plane displacement and longitudinal contribution to stroke volume (SV long\% $_{2}$. CMR images in 2-chamber (2CH), 3-chamber $(3 \mathrm{CH})$, 4-chamber $(4 \mathrm{CH})$, and short-axis $(\mathrm{SA})$ views at end diastole (ED, upper panes) and end systole (ES, lower panes). The solid lines indicate the atrioventricular planes at ED and the dashed lines at ES. The atrioventricular plane displacement (AVPD) is defined as the distance the

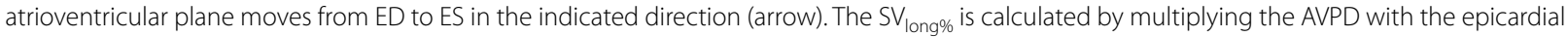
short-axis area in ED (marked area in the SA view at the level of the dashed lines) comprising the atrioventricular plane motion, divided with the left ventricular stroke volume
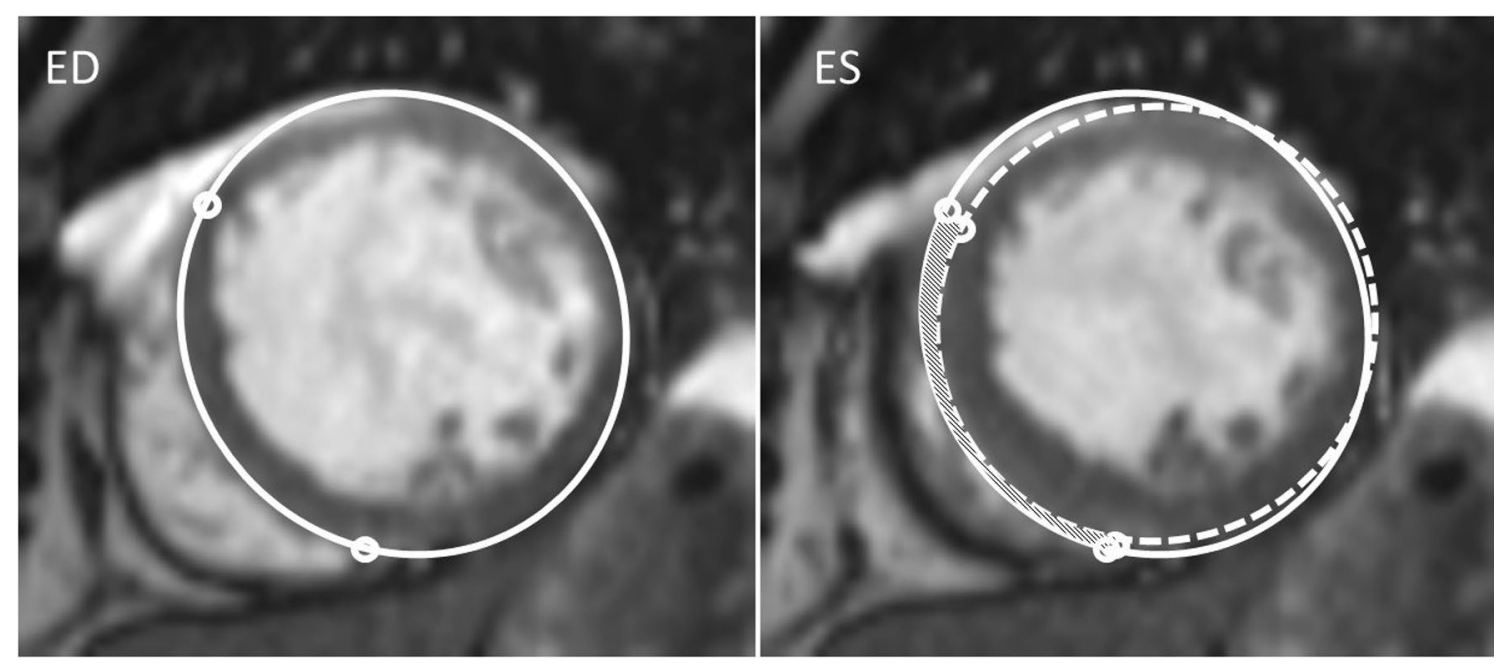

Fig. 3 Septal and lateral contribution to stroke volume $\left(S V_{\text {sept } \%}\right.$ and $\left.S V_{\text {lat } \%}\right)$. Short-axis views in end-diastole (ED, left pane) and end-systole (ES, right pane). Left ventricular (LV) epicardial border in ED is represented by the solid line. LV epicardial border in ES is represented by the dashed line. Right ventricular (RV) insertion points are represented as white circles. $\mathrm{SV}_{\text {sept\% }}$ is measured with the area defined by the RV insertion points and the septal borders in ED and ES (marked area). SV lat\% is measured with the area defined by the lateral LV borders between ED and ES, excluding the septum

Echocardiographic assessment of LV volumes was analyzed in EchoPac BT12 (GE Medical, Horten, Norway) offline. Patients were examined prior to and six months after CRT implantation in accordance with study protocol [8].

The effect of CRT was evaluated with echocardiography at baseline prior to CRT implantation and at six 
months follow-up after implantation to assess reverse remodeling. Response to CRT was defined as previously proposed [22] as $\geq 15 \%$ reduction in LVESV measured by the Simpson's biplane method [23]. Dyssynchrony was assessed at baseline and was defined as anteroseptal to posterior mid-left ventricular delay $\geq 130 \mathrm{~ms}$ using radial speckle-tracking analysis as previously described [7].

\section{Statistical analysis}

Statistical analyses were performed using IBM SPSS version 25 (SPSS inc., Chicago, Illinois, USA).

Continuous variables are expressed as mean \pm SD. Normal distribution was determined by visual assessment of histograms. For $\mathrm{SV}_{\text {sept\% }}$, the cutoff value was $0 \%$, with negative contribution to LVSV as a sign of cardiac dyssynchrony [24]. Groups were compared with unpaired student's t-tests for continuous variables and Pearson's chi-squared test or Fisher's exact test for categorical variables. Individual baseline and follow-up data were compared using paired student's t-tests. Results with $P$ values $<0.05$ were considered statistically significant. Univariable and multivariable logistic regression analyses were conducted with responder as dependent variable and $\mathrm{SV}_{\text {long\%, }} \mathrm{SV}_{\text {lat\%}}$, and $\mathrm{SV}_{\text {sept\% }}$ as predictors. Demographic, clinical, and echocardiographic predictors were added to the model to adjust for confounding factors. Candidate predictors in the univariable logistic analysis that had a $P$ value of $<0.25$ were included in the multivariable analysis [25]. Included predictors were retained in the model if $P<0.05$. Post-hoc sample size analysis was conducted using the software PS: Power and Sample Size Calculation (version 3.1.6, October 2018) [26]. Assumptions included alpha level of 0.05 , statistical power of 0.8 , and effect size and standard deviations from the present study.

\section{Results}

\section{Study population}

Sixty-five patients fulfilled the study protocol. Nineteen were women, mean age was $67 \pm 9$ years. $51 \%$ had nonischemic etiology and QRS duration on standard ECG was $167 \pm 17$ ms. Mean ejection fraction was $26 \pm 8 \%$, and $86 \%$ had typical LBBB. The characteristics of the cohort are in detail presented in Table 1.

\section{Regional contributions in CRT candidates and healthy controls}

In patients receiving $\mathrm{CRT}$, the relative regional contributions to LVSV were for $\mathrm{SV}_{\text {long\% }} 53 \pm 18 \%$, for $\mathrm{SV}_{\text {lat\% }}$ $41 \pm 16 \%$, and for $\mathrm{SV}_{\text {sept\% }} 0 \pm 15 \%$. Negative (abnormal) $\mathrm{SV}_{\text {sept\% }}$ was found in $43 \%$ of the patients eligible for CRT. Thus, just above half of the selected CRT candidates did
Table 1 Baseline patient characteristics

\begin{tabular}{|c|c|c|}
\hline & Patients $(n=65)$ & Controls $(n=20)$ \\
\hline Women & 19 (29\%) & $8(40.0 \%)$ \\
\hline Age (years) & $67 \pm 9^{\dagger}$ & $62 \pm 11$ \\
\hline $\mathrm{BSA}\left(\mathrm{m}^{2}\right)$ & $2.0 \pm 0.2^{\dagger}$ & $1.9 \pm 0.2$ \\
\hline Diabetes & $9(14 \%)$ & 0 \\
\hline QRS-duration (ms) & $167 \pm 17^{\dagger+\dagger}$ & $95 \pm 11$ \\
\hline LBBB & $56(86 \%)$ & N/A \\
\hline Heart rate (beats/min) & $67 \pm 13^{+}$ & $62 \pm 7$ \\
\hline \multicolumn{3}{|l|}{$\mathrm{NIBP}(\mathrm{mmHg})$} \\
\hline Systolic & $127 \pm 17$ & $131 \pm 13$ \\
\hline Diastolic & $76 \pm 9$ & $75 \pm 7$ \\
\hline Medication & & 0 \\
\hline ACEI/ARB & $65(100 \%)$ & \\
\hline Betablocker & $58(89 \%)$ & \\
\hline Diuretics & $56(86 \%)$ & \\
\hline Antihyperlipidemics & $41(63 \%)$ & \\
\hline Platelet aggregation inhibitors & $35(54 \%)$ & \\
\hline NYHA Class & & N/A \\
\hline NYHA Class II & $20(31 \%)$ & \\
\hline NYHA Class III & $41(63 \%)$ & \\
\hline NYHA Class IV & $4(6 \%)$ & \\
\hline \multicolumn{3}{|l|}{ Etiology } \\
\hline Ischemic & $32(49 \%)$ & \\
\hline Non-ischemic & $33(51 \%)$ & \\
\hline LVEDV $(\mathrm{ml})$ & $326 \pm 115^{t+t}$ & $163 \pm 37$ \\
\hline LVESV (ml) & $246 \pm 110^{\dagger+t}$ & $66 \pm 21$ \\
\hline LVSV (ml) & $80 \pm 23^{t+}$ & $97 \pm 20$ \\
\hline LVEF (\%) & $26 \pm 8^{\dagger+\dagger}$ & $60 \pm 5$ \\
\hline LV mass (g) & $170 \pm 49^{t+\dagger}$ & $112 \pm 32$ \\
\hline CO (I/min) & $5.3 \pm 1.5^{\dagger}$ & $6.0 \pm 1.2$ \\
\hline LVAVPD (mm) & $8.3 \pm 3.2^{+t \dagger}$ & $15.3 \pm 1.6$ \\
\hline SA area $\left(\mathrm{cm}^{2}\right)$ & $51 \pm 11^{t+\dagger}$ & $34 \pm 7$ \\
\hline$S V_{\text {long\% }}(\%)$ & $53 \pm 18^{\dagger+}$ & $64 \pm 8$ \\
\hline $\mathrm{SV}_{\text {lat} \%}(\%)$ & $41 \pm 16^{t+\dagger}$ & $29 \pm 7$ \\
\hline $\mathrm{SV}_{\text {sept\% }}(\%)$ & $0 \pm 15^{t+t}$ & $10 \pm 4$ \\
\hline LGE positive & $40(62 \%)$ & N/A \\
\hline
\end{tabular}

Continuous variables are expressed as means \pm SD and categorical values in absolute numbers and proportion in parenthesis

ACEl, angiotensin-converting enzyme inhibitor; ARB, angiotensin II receptor blocker; $\mathrm{BSA}$, body surface area; $\mathrm{CO}$, cardiac output; $\mathrm{DCM}$, dilated cardiomyopathy; IHD, ischemic heart disease; LBBB, left bundle branch block; LV, left ventricular; $A V P D$, atrioventricular plane displacement; $E D V$, left ventricular end-diastolic volume; EF, left ventricular ejection fraction; ESV, left ventricular end-systolic volume; SV, left ventricular stroke volume; NCC, non-compaction cardiomyopathy; NIBP, non-invasive systemic blood pressure; NYHA, New York Heart Association; $\mathrm{SV}_{\text {lat\% }}$ l lateral contribution to stroke volume; $\mathrm{SV}_{\text {long\% }}$ longitudinal contribution to stroke volume; $\mathrm{SV}_{\text {sept\%, }}$, septal contribution to stroke volume

${ }^{+} P<0.05$ patients vs healthy controls

${ }^{+\dagger} P<0.01$ patients vs healthy controls

${ }^{+t+} P<0.001$ patients vs healthy controls 
not have a septal net movement towards the right ventricle during systole.

Compared to controls, the longitudinal shortening expressed as AVPD $(8.3 \pm 3.2$ vs $15.3 \pm 1.6 \mathrm{~mm}, P<0.001)$ and $\mathrm{SV}_{\text {long\% }}$ were lower in patients $(53 \pm 18$ vs $64 \pm 8 \%$, $P<0.01)$. Also, patients had a lower $\mathrm{SV}_{\text {sept\% }}(0 \pm 15 \%$ vs $10 \pm 4 \%, P<0.001)$ accompanied with an increase in $\mathrm{SV}_{\text {lat } \%}(41 \pm 16 \%$ vs $29 \pm 7 \%, P<0.001)$. Absolute SV was lower $(80 \pm 23 \mathrm{ml}$ vs $97 \pm 20 \mathrm{ml}, P<0.01)$ and the shortaxis area was larger in patients compared to controls $\left(51 \pm 11 \mathrm{~cm}^{2}\right.$ vs $\left.34 \pm 7 \mathrm{~cm}^{2}, P<0.001\right)$ (Table 1$)$.

\section{Outcome}

At six months follow-up echocardiography, LVESV was decreased $\geq 15 \%$ in 37 patients (57\%), as an objective marker of reverse remodeling and a positive response to CRT. The mean $\triangle \mathrm{LVESV}$ was $-18 \pm 22 \%(P<0.001$, Fig. 4). Tables 2 and 3 show the ability of each individual proposed CMR predictor to stratify patients according to reverse remodeling response. There were no differences in $\mathrm{SV}_{\text {long\% }}, \mathrm{SV}_{\text {lat\%}}$, nor $\mathrm{SV}_{\text {sept\% }}$ between $\mathrm{CRT}$ responders and non-responders (Table 3 ).

Forty-six patients (71\%) improved in NYHA functional classification $\geq 1$ at six months followup. Twenty-seven out of 63 patients (43\%) had

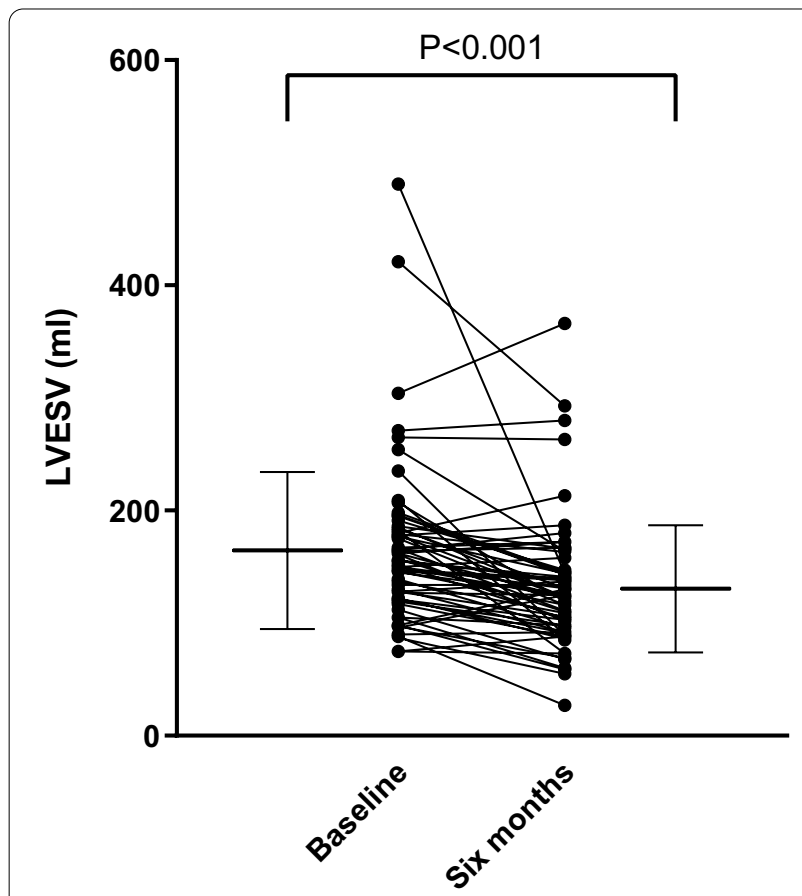

Fig. 4 Illustration of reverse remodeling. Paired individual values at baseline and six months follow-up. Error bars denote mean values $\pm S D$. There was a significant reduction in left ventricular end-systolic volume (LVESV) by echocardiography six months after CRT implantation
Table 2 Comparison of patients with negative and positive septal contribution to stroke volume

\begin{tabular}{|c|c|c|c|c|c|}
\hline $\begin{array}{l}\text { CMR } \\
\text { parameter }\end{array}$ & $\begin{array}{l}\Delta \text { LVESV } \geq 15 \% \\
(n=37)\end{array}$ & $\begin{array}{l}\Delta \text { LVESV }<15 \% \\
(n=28)\end{array}$ & $P$ & $\begin{array}{l}\Delta \text { LLVESV } \\
(\%)\end{array}$ & $P$ \\
\hline $\begin{array}{l}\text { Negative } \\
\text { SV }_{\text {sept } \%}\end{array}$ & 16 & 12 & 0.98 & $-18 \pm 22$ & 0.96 \\
\hline $\begin{array}{l}\text { Positive } \\
\text { SV }_{\text {sept } \%}\end{array}$ & 21 & 16 & & $-19 \pm 23$ & \\
\hline
\end{tabular}

dyssynchrony on echocardiography at baseline. Two patients were not assessable for dyssynchrony analysis. There were no associations between dyssynchrony and CRT outcomes, defined as LVESV reduction $\geq 15 \%$ $(P=0.66)$ and NYHA functional classification improved $\geq 1(P=0.47)$.

Multivariable logistic regression to study the effect of $\mathrm{SV}_{\text {long\% }}, \mathrm{SV}_{\text {lat\%}}$, and $\mathrm{SV}_{\text {sept\% }}$ on LVESV response was not significant $(P=0.17)$. When demographic, clinical, and echocardiographic variables were added to account for confounding to the model, the model remained nonsignificant $\left(P=0.10\right.$, Table 4). Further, $\mathrm{SV}_{\text {long\% }}, \mathrm{SV}_{\text {lat } \% \text {, }}$ and $\mathrm{SV}_{\text {sept\% }}$ did not predict outcome defined as NYHA response (defined as improvement in $\geq 1$ NYHA classification at six months follow-up, Table 5).

\section{Ischemic versus non-ischemic}

Patients with ischemic and non-ischemic etiology of heart failure were compared in a sub-group analysis of the patient characteristics (Table 6). Patients with ischemic heart failure were older, had more platelet aggregation inhibitor treatment and more LGE, but did not otherwise differ from the non-ischemic patients. Both groups had similar QRS-duration $(167 \pm 20$ vs $167 \pm 13 \mathrm{~ms}, P=0.96)$ and proportion of LBBB $(81 \%$ vs $91 \%, P=0.26)$. Out of the 33 patients with nonischemic etiology of heart failure, there were more responders than non-responders to CRT ( $\mathrm{n}=23$ vs $\mathrm{n}=10, P<0.05)$. There was no difference in reverse remodeling between patients with ischemic heart disease compared to patients with non-ischemic heart disease ( $\triangle$ LVESV $-14 \pm 20 \mathrm{ml}$ vs $-23 \pm 24 \mathrm{ml}, P=0.12$ ). Longitudinal shortening did not differ in patients with ischemic compared to non-ischemic etiology (AVPD $7.7 \pm 3.0 \mathrm{~mm}$ vs $8.9 \pm 3.2 \mathrm{~mm}, P=0.15)$. There were no differences in regional contributions to SV between patients with ischemic vs non-ischemic heart disease (Table 6). 
Table 3 Comparison of regional contributions to SV in responders and non-responders to CRT

\begin{tabular}{|c|c|c|c|}
\hline & $\begin{array}{l}\text { Responders } \\
\Delta \text { LVESV } \geq 15 \%(n=37)\end{array}$ & $\begin{array}{l}\text { Non-responders } \\
\Delta \mathrm{LVESV}<15 \%(\mathrm{n}=28)\end{array}$ & $P$ \\
\hline LVAVPD (mm) & $8.0 \pm 3.1$ & $8.8 \pm 3.2$ & 0.31 \\
\hline $\mathrm{SV}_{\text {long } \%}(\%)$ & $53 \pm 18$ & $53 \pm 19$ & 0.87 \\
\hline $\mathrm{SV}_{\text {lat } \%}(\%)$ & $38 \pm 15$ & $45 \pm 16$ & 0.09 \\
\hline $\mathrm{SV}_{\text {sept} \%}(\%)$ & $-1 \pm 17$ & $1 \pm 11$ & 0.65 \\
\hline LGE positive & $23(62 \%)$ & $17(61 \%)$ & 0.91 \\
\hline Non-ischemic/ischemic heart disease & $23(62 \%) / 14(38 \%)$ & $10(36 \%) / 18(64 \%)$ & $<0.05$ \\
\hline $\begin{array}{l}\text { Anterioseptal to posterior mid-left ventricular } \\
\text { delay } \geq 130 \mathrm{~ms}\end{array}$ & $15(41 \%)$ & $12(46 \%)$ & 0.66 \\
\hline QRS duration $\geq 150 \mathrm{~ms}$ & $34(92 \%)$ & $23(82 \%)$ & 0.28 \\
\hline LBBB & $34(92 \%)$ & $22(79 \%)$ & 0.16 \\
\hline Male/female sex & $27(73 \%) / 10(27 \%)$ & $19(68 \%) / 9(32 \%)$ & 0.65 \\
\hline
\end{tabular}

Responders vs non-responders. Response is defined as a negative change in left-ventricular end-systolic volume ( $\Delta$ LVESV) of $\geq 15 \%$ by echocardiography. Values are expressed as means \pm SD or absolute number and proportion in parenthesis

CRT: cardiac resynchronization therapy; LBBB: left bundle branch block; LVAVPD: left ventricular atrioventricular plane displacement; SV ${ }_{\text {long\%: }}$ longitudinal contribution

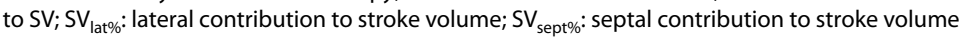

Table 4 Logistic regression analysis with LVESV response as dependent variable

\begin{tabular}{|c|c|c|c|c|c|c|}
\hline \multirow[t]{2}{*}{ Dependent variable: LVESV response } & \multicolumn{3}{|c|}{ Univariable } & \multicolumn{3}{|c|}{ Multivariable } \\
\hline & OR & $95 \% \mathrm{Cl}$ & $P$ & OR & $95 \% \mathrm{Cl}$ & $P$ \\
\hline $\begin{array}{l}\text { Anteroseptal to posterior mid-left ventricular } \\
\text { delay } \geq 130 \mathrm{~ms}\end{array}$ & 1.26 & $0.46-3.46$ & 0.66 & - & - & - \\
\hline QRS duration $\geq 150 \mathrm{~ms}$ & 2.46 & $0.54-11.33$ & 0.25 & 2.41 & $0.45-13.01$ & 0.31 \\
\hline LBBB & 3.09 & $0.70-13.66$ & 0.14 & 2.25 & $0.46-11.01$ & 0.32 \\
\hline Ischemic Heart Disease & 0.34 & $0.12-0.94$ & 0.04 & 0.46 & $0.16-1.37$ & 0.16 \\
\hline Male sex & 1.28 & $0.44-3.75$ & 0.65 & - & - & - \\
\hline $\mathrm{SV}_{\text {long } \%}$ & 1.00 & $0.98-1.03$ & 0.86 & - & - & - \\
\hline$S V_{\text {lat } \%}$ & 0.97 & $0.94-1.01$ & 0.09 & 0.98 & $0.94-1.01$ & 0.17 \\
\hline $\mathrm{SV}_{\text {sept} \%}$ & 0.99 & $0.96-1.03$ & 0.64 & - & - & - \\
\hline
\end{tabular}

Univariable and multivariable logistic regression analyses. Significant $\mathrm{P}$ values in the univariable analysis (cut-off $<0.25)$ were included in the multivariable analysis and are indicated in bold

LBBB, left bundle branch block; LVESV: left ventricular end-systolic volume; $\mathrm{SV}_{\text {long} \%}$ : longitudinal contribution to SV; $\mathrm{SV}_{\text {lat } \%}$ : lateral contribution to 5 troke volume; $\mathrm{SV}_{\text {sept} \%}$ : septal contribution to stroke volume

\section{Post-hoc sample size analysis}

There were no significant differences in LVAVPD, $\mathrm{SV}_{\text {long\%}}, \mathrm{SV}_{\text {lat\%}}$, and $\mathrm{SV}_{\text {sept\% }}$ between responders and non-responders as the $95 \%$ confidence intervals for the two groups overlapped for all parameters. This study could have yielded false-negative results (i.e. type II error) due to too few participants. To investigate the risk of type II error, a post-hoc sample size analysis was performed. Sample sizes of $n=342, n=3975$, $\mathrm{n}=96$ and $\mathrm{n}=4362$ respectively for LVAVPD, $S V_{\text {long\%, }}$, $\mathrm{SV}_{\text {lat\%}}$, and $\mathrm{SV}_{\text {sept\% }}$ would have been needed to detect a signal.

\section{Discussion}

This study shows that patients eligible for CRT treatment have both lower longitudinal systolic atrioventricular plane displacement and septal contribution to SV, compared to healthy controls. However, patients have higher lateral contribution to SV, which is interpreted as a compensatory mechanism to the decreased septal contribution. While this is the first study showing these differences between patients with HF and electromechanical dyssynchrony and healthy controls, the suggested measures of regional function could not predict CRT outcome in terms of reverse remodeling at 
Table 5 Logistic regression analysis with NYHA response as dependent variable

\begin{tabular}{|c|c|c|c|c|c|c|}
\hline \multirow[t]{2}{*}{ Dependent variable: NYHA response } & \multicolumn{3}{|c|}{ Univariable } & \multicolumn{3}{|c|}{ Multivariable } \\
\hline & OR & $95 \% \mathrm{Cl}$ & $P$ & OR & $95 \% \mathrm{Cl}$ & $P$ \\
\hline $\begin{array}{l}\text { Anteroseptal to posterior mid-left ventricular } \\
\text { delay } \geq 130 \mathrm{~ms}\end{array}$ & 1.50 & $0.50-4.50$ & 0.47 & - & - & - \\
\hline QRS duration $\geq 150 \mathrm{~ms}$ & 1.54 & $0.33-7.20$ & 0.59 & - & - & - \\
\hline LBBB & 1.25 & $0.28-5.62$ & 0.77 & - & - & - \\
\hline Ischemic Heart Disease & 1.5 & $0.51-4.41$ & 0.46 & - & - & - \\
\hline Male sex & 0.82 & $0.25-2.71$ & 0.74 & - & - & - \\
\hline$S V_{\text {long } \%}$ & 0.98 & $0.95-1.01$ & 0.23 & - & - & - \\
\hline $\mathrm{SV}_{\text {lat} \%}$ & 1.00 & $0.97-1.03$ & 0.99 & - & - & - \\
\hline $\mathrm{SV}_{\text {sept } \%}$ & 1.00 & $0.96-1.03$ & 0.79 & - & - & - \\
\hline
\end{tabular}

Univariable and multivariable logistic regression analyses. Significant $\mathrm{P}$ values in the univariable analysis (cut-off $<0.25$ ) were included in the multivariable analysis LBBB, left bundle branch block; NYHA: New York Heart Association; SV long\%: longitudinal contribution to SV; SV $_{\text {lat } \%}$ : lateral contribution to stroke volume; $\mathrm{SV}_{\text {sept } \%}$ : Septal contribution to stroke volume

six months follow-up. In non-ischemic cardiomyopathy, reverse remodeling was more common compared to ischemic disease. Despite this, no difference in regional contribution to SV could be seen between ischemic and non-ischemic patients. Even when assessing with multivariable analysis of demographic, clinical and CMR characteristics, there was no association with responding on CRT treatment in the CMR parameters. Thus, our study is in line with previous studies that could not show any added value of regional function measures to further decrease the number of non-responders in patients selected for CRT treatment compared to current guidelines $[4,27]$.

\section{Search for novel prognostic predictors of response to CRT}

As of today, presence of LBBB, non-ischemic etiology, sex, QRS-duration, and sinus rhythm are the only factors that have been found to consistently predict response to CRT in randomized prospective trials [28]. Mechanical measures of dyssynchrony have been identified by echocardiography, for example the retrospective cohort study PREDICT-CRT trial which showed that presence of septal flash and apical rocking on echocardiography were markers of mechanical dyssynchrony [29]. However, randomized trials are warranted before new clinical guidelines of patient selection to CRT can be implemented. Other markers of mechanical and electrical dyssynchrony (e.g. left ventricular pre-ejection period, inter- and intraventricular delay by tissue Doppler imaging, and systolic stretch index) have also been investigated using echocardiography [30, 31], but with limited success. A novel approach with echocardiography is to determine wasted septal work with regional pressure-strain loops [11] and this was shown in retrospective data to predict outcome together with septal flash [32]. Mechanical measures of dyssynchrony have also been described in patients with LBBB using CMR [24]. Further, myocardial scar in the paced segment affect CRT effect negatively [33], and scar has been shown in a retrospective observational study to independently predict clinical events and LV functional improvement [34]. However, prospective randomized controlled trials are needed to validate imaging measures as predictors of CRT.

The present prospective observational study aimed to test if longitudinal and septal movements quantified by CMR can predict response to CRT. Longitudinal function defined as AVPD and $\mathrm{SV}_{\text {long\% }}$ was lower in patients eligible for CRT compared to healthy controls. Even if decreased AVPD carries prognostic information of poor outcome in a multitude of diseases, longitudinal function defined as AVPD and $\mathrm{SV}_{\text {long\% }}$ was not related to treatment response in CRT-eligible patients.

In patients referred for CRT, we found a net inverse motion of the septum and thus negative contribution to SV in almost half the patients. This contrasted to healthy controls who all had a positive contribution to SV. However, there was no difference in reverse remodeling 6 months after treatment between patients with negative vs positive septal contribution to LVSV. One explanation for the heterogenous response to CRT may be that differences in regions affected by disease or presence of LBBB give rise to different patterns of mechanical dyssynchrony [24]. Electrical dyssynchrony is not necessarily accompanied with mechanical dyssynchrony susceptible for CRT [35]. Another possible explanation for the lack of response in patients with negative $\mathrm{SV}_{\text {sept\% }}$ is that the measurement was not time resolved. Abnormal septal contractions happening before and after endsystole might therefore have been missed. Post-systolic contraction/shortening is an inefficient contraction after 
Table 6 Subgroup analysis of patients with ischemic and non-ischemic heart disease

\begin{tabular}{|c|c|c|c|}
\hline & Ischemic heart disease $(n=32)$ & Non-ischemic heart disease $(n=33)$ & $P$ \\
\hline Women & $6(19)$ & $13(39)$ & 0.07 \\
\hline Age (years) & $71 \pm 8$ & $63 \pm 8$ & $<0.001$ \\
\hline $\mathrm{BSA}\left(\mathrm{m}^{2}\right)$ & $1.97 \pm 0.19$ & $2.03 \pm 0.24$ & 0.26 \\
\hline Diabetes & $4(13)$ & $5(15)$ & 1.0 \\
\hline QRS-duration (ms) & $167 \pm 20$ & $167 \pm 13$ & 0.96 \\
\hline LBBB & $26(81)$ & $30(91)$ & 0.30 \\
\hline Heart rate (beats/min) & $68 \pm 15$ & $67 \pm 10$ & 0.87 \\
\hline \multicolumn{4}{|l|}{$\mathrm{NIBP}(\mathrm{mmHg})$} \\
\hline Systolic & $128 \pm 18$ & $127 \pm 16$ & 0.75 \\
\hline Diastolic & $75 \pm 8$ & $77 \pm 9$ & 0.47 \\
\hline \multicolumn{4}{|l|}{ Medication } \\
\hline ACEI/ARB & $32(100)$ & $33(100)$ & 1.0 \\
\hline Betablocker & $28(88)$ & $30(91)$ & 0.66 \\
\hline Diuretics & $27(84)$ & $29(88)$ & 0.68 \\
\hline Antihyper-lipidemics & $29(91)$ & $12(36)$ & \\
\hline Platelet aggregation inhibitors & $24(75)$ & $11(33)$ & 0.001 \\
\hline NYHA Class & & & 0.32 \\
\hline NYHA Class II & $8(25)$ & $12(36)$ & \\
\hline NYHA Class III & $23(72)$ & $18(55)$ & \\
\hline NYHA Class IV & $1(3)$ & $3(9)$ & \\
\hline \multicolumn{4}{|l|}{ Echocardiagraphy } \\
\hline$\Delta \operatorname{LVESV}(\%)$ & $-14 \pm 20$ & $-23 \pm 24$ & 0.12 \\
\hline \multicolumn{4}{|l|}{ CMR } \\
\hline $\mathrm{EDV}(\mathrm{ml})$ & $323 \pm 68$ & $329 \pm 148$ & 0.82 \\
\hline ESV (ml) & $242 \pm 61$ & $250 \pm 143$ & 0.77 \\
\hline $\mathrm{SV}(\mathrm{ml})$ & $80 \pm 26$ & $79 \pm 21$ & 0.80 \\
\hline$E F(\%)$ & $25 \pm 7$ & $27 \pm 9$ & 0.40 \\
\hline $\mathrm{CO}(1 / \mathrm{min})$ & $5.3 \pm 1.6$ & $5.3 \pm 1.5$ & 0.90 \\
\hline LVAVPD (mm) & $7.7 \pm 3.0$ & $8.9 \pm 3.2$ & 0.15 \\
\hline $\mathrm{SV}_{\text {long }} \%$ & $49 \pm 17$ & $57 \pm 19$ & 0.10 \\
\hline$S V_{\text {lat } \%}$ & $45 \pm 18$ & $37 \pm 13$ & 0.07 \\
\hline$S V_{\text {sept } \%}$ & $-1 \pm 14$ & $2 \pm 16$ & 0.41 \\
\hline LGE positive & $28(88 \%)$ & $12(36 \%)$ & $<0.01$ \\
\hline
\end{tabular}

Values are expressed as means \pm SD or in absolute number and proportion in parenthesis. $\triangle L V E S V$, change in left ventricular end-systolic volume from pre to 6 months post CRT echocardiography

$\mathrm{ACEl}$, angiotensin-converting enzyme inhibitor; ARB, angiotensin II receptor blocker; BSA, body surface area; $C O$, cardiac output; CMR, cardiac magnetic resonance imaging, EDV, end-diastolic volume; EF, ejection fraction; ESV, end-systolic volume; LBBB, left bundle branch block; LVAVPD, left ventricular atrioventricular plane displacement; NIBP, non-invasive systemic blood pressure; NYHA, New York Heart Association; SV, stroke volume; SVlong\%, longitudinal contribution to SV; SVlat\%, lateral contribution to stroke volume; SVsept\%, septal contribution to stroke volume

aortic valve closure and has recently gained attention as a potential diagnostic predictor [36]. It is possible that post-systolic contraction reflects cardiac mechanical dyssynchrony susceptible to CRT.

Guiding of CRT using a multi-modality imaging strategy, with a combination of cardiac venous anatomy using cardiac computer tomography, myocardial perfusion using single-photon emission computed tomography, and deformation using speckle-tracking echocardiography, showed improvement of a composite clinical outcome in a prospective randomized controlled single-center trial [6]. Also, combining dyssynchrony and fibrosis data using CMR in a cohort study of 40 patients showed a sensitivity of $90 \%$ and specificity of $58 \%$ to identify clinical responders [37]. However, in our prospective randomized CRT study we could not improve the degree of reverse remodeling at 6 months when guiding electrode placements using a multi-modality approach of strain data from echocardiography, venography from computer tomography, and fibrosis from CMR [15]. The neutral results 
from the main study allowed us to perform this sub-study without taking into account if patients were randomized to image-guided electrode placement or not.

\section{Defining response to CRT}

The response rate to CRT is dependent on the selected outcome measure and study population characteristics [38]. In this study, we chose $\triangle$ LVESV from echocardiography at six months follow-up as a measure to define reverse remodeling as the primary outcome. This is a surrogate marker that has been shown to be reliable for CRT response [39]. The reverse remodeling response with reduced $\triangle \mathrm{LVESV}$ to CRT was in accordance to previous studies [40, 41], both regarding mean $\triangle$ LVESV and proportion of responders defined as $\geq 15 \%$ LVESV reduction after six months. Alternative outcome variables to physiological measures commonly used to evaluate HF progression include clinical assessment, major adverse cardiac events, hospitalization and mortality [42]. However, our patient cohort was too small for such an event evaluation according to the post-hoc sample size analysis.

\section{Future perspective}

The present study investigated the ability of a novel functional measure using CMR to predict outcome after CRT. Regional contributions to SV with this method were not sufficient in their prognostic ability, despite showing decreased longitudinal and septal function with a relatively increased lateral contribution function when compared with healthy volunteers. Currently, there is no single predictor of response to CRT, reflecting a complex syndrome with multifactorial causes. Recently a multicenter study showed that integrating multiple variables such as wasted work from echocardiography strain in combination with septal viability from CMR had the best capability to identify CRT responders [43]. Therefore, future strategies may move from simpler measures of function such as EF and myocardial dyssynchrony to more advanced combination of functional and structural measures. Which image modalities that will provide the aforementioned information remain to be determined in prospective randomized trials, and whether CMR can provide the same information as echocardiography remains to be shown.

\section{Limitations}

CMR exams at follow-up would have been preferred to echocardiography, but CMR-compatible devices were not clinically available at the course of the study. However, all echocardiographic exams were performed by one sonographer ( $>15$ years of experience) at both inclusion and at follow-up, and all analyses were performed by one expert reader ( $>25$ years of experience) at a tertiary highvolume center.

The study group consisted of a heterogenous HF population: $49 \%$ of study participants had ischemic etiology, a group that has been shown to have a higher non-response rate to CRT than patients with non-ischemic heart failure [33, 44]. Subgroup analysis comparing ischemic HF to non-ischemic HF in this study did not show any difference in reverse remodeling between the two etiologies. Furthermore, $\mathrm{SV}_{\text {long\% }}, \mathrm{SV}_{\text {lat\%, }}$, and $\mathrm{SV}_{\text {sept\% }}$ were affected similarly in ischemic and non-ischemic HF with a trend towards lower $\mathrm{SV}_{\text {long\% }}$ and higher $\mathrm{SV}_{\text {lat\% }}$ in ischemic etiology, albeit not statistically significant.

The CMR protocol in the present study used LGE for tissue characterization. Since the conceptualization of the present study, T1 mapping and extra cellular volume index (ECV) measurements have become a standard part of heart failure evaluation with CMR, providing added prognostic value in non-ischemic heart failure $[45,46]$. In the context of CRT selection, focal scar burden using LGE is associated with worse outcome, but diffuse scar burden using $\mathrm{T} 1$ mapping do not independently predict CRT outcome [47].

The post-hoc sample size analysis resulted in larger sample sizes than in the present study. Assuming there are true differences between responders and nonresponders, they are probably small and would not be of clinical relevance to facilitate the selection of CRT responders on an individual level.

\section{Conclusions}

Quantitative CMR assessment shows that patients eligible for CRT have decreased longitudinal function and that only half of the patients have a net shift of the septum towards the right ventricle during systole despite the majority of patients had LBBB. However, responders with reverse LV remodeling do not differ from non-responders regarding the longitudinal, lateral, nor septal contribution to SV. Patients with ischemic HF did not differ in regional contributions to SV compared to patients with non-ischemic HF. Thus, these regional contributions to $\mathrm{SV}$ are not applicable as predictors of LV remodeling in patients with LBBB eligible for CRT.

\footnotetext{
Abbreviations

AVPD: Atrioventricular plane displacement; CMR: Cardiac magnetic resonance imaging; CRT: Cardiac resynchronization therapy; EDV: End-diastolic volume; EF: Ejection fraction; ESV: End-systolic volume; HF: Heart failure; LGE: Late gadolinium enhancement; LV: Left ventricle; NYHA: New York Heart Association; RV: Right ventricle; SV: Stroke volume; SV ${ }_{1+0}$ : Lateral contribution to stroke volume; $\mathrm{SV}_{\text {long\%: }}$ Longitudinal contribution to stroke volume; $\mathrm{SV}_{\text {sept } \%}$ : Septal contribution to stroke volume.
}

\section{Acknowledgements}

None. 


\section{Authors' contributions}

BÖ analyzed and interpreted CMR, subject characteristics data and drafted the manuscript. EO and MC acquired and analyzed CMR data. AWE and AR performed and analyzed echocardiography examinations. ZB and RB acquired clinical patient data. MK analyzed CMR data. RB and MC conceptualized and designed the study. All authors read and approved the final manuscript.

\section{Funding}

Open access funding provided by Lund University. This study was funded by the Swedish Research Council, Swedish Heart Lung Foundation, Wallenberg Centre for Molecular Medicine at Lund University, ALF Medical Faculty Grants at Lund University. The funding agencies have not had any influence on the design of the study or interpretation of the results.

\section{Availability of data and materials}

The datasets generated during and/or analyzed during the current study are available from the corresponding author on reasonable request.

\section{Declarations}

\section{Ethics approval and consent to participate}

The study was performed in accordance with the Declaration of Helsinki. The regional department of the Swedish Ethical Review Authority, Lund, Sweden, approved the study and all study participants provided written informed consent prior to examinations. Reference number: Dnr 2010/380.

\section{Consent for publication}

Not applicable.

\section{Competing interests}

The authors declare that they have no competing interests.

\section{Author details}

${ }^{1}$ Clinical Physiology, Department of Clinical Sciences Lund, Lund University, Skåne University Hospital, Lund, Sweden. ${ }^{2}$ Section for Heart Failure and Valvular Disease, Department of Clinical Sciences Lund, Cardiology, Lund University, Skåne University Hospital, Lund, Sweden. ${ }^{3}$ Section of Arrhythmia, Department of Clinical Sciences Lund, Cardiology, Lund University, Skåne University Hospital, Lund, Sweden. ${ }^{4}$ Department of Radiology, New York University School of Medicine, New York, NY, USA. ${ }^{5}$ Wallenberg Center for Molecular Medicine, Lund University, Lund, Sweden.

Received: 14 August 2020 Accepted: 15 October 2021

Published online: 26 October 2021

\section{References}

1. Sweeney $\mathrm{MO}$, Prinzen FW. Ventricular pump function and pacing: physiological and clinical integration. Circ Arrhythm Electrophysiol. 2008;1(2):127-39.

2. Piotr Ponikowski Voors AA, Anker SD, Cleland JGF, Uk AJSC, Harjola V Germany VF, et al. 2016 ESC Guidelines for the diagnosis and treatment of acute and chronic heart failure The Task Force for the diagnosis and treatment of acute and chronic heart failure of the European Society of Cardiology (ESC) developed with the special contribution. Eur Heart J. 2016:128(20):1-85.

3. Cleland JGF, Daubert J-C, Erdmann E, Freemantle N, Gras D, Kappenberger $\mathrm{L}$, et al. The effect of cardiac resynchronization on morbidity and mortality in heart failure. N Engl J Med. 2005;352(15):1539-49.

4. Chung ES, Leon AR, Tavazzi L, Sun JP, Nihoyannopoulos P, Merlino J, et al. Results of the predictors of response to CRT (PROSPECT) trial. Circulation. 2008;117(20):2608-16.

5. Ruschitzka F, Abraham WT, Singh JP, Bax JJ, Borer JS, Brugada J, et al. Cardiac-resynchronization therapy in heart failure with a narrow QRS complex. N Engl J Med. 2013;369(15):1395-405.

6. Sommer A, Kronborg MB, Nørgaard BL, Poulsen SH, Bouchelouche K, Böttcher $\mathrm{M}$, et al. Multimodality imaging-guided left ventricular lead placement in cardiac resynchronization therapy: a randomized controlled trial. Eur J Heart Fail. 2016;18(11):1365-74.
7. Borgquist R, Carlsson M, Markstad H, Werther-Evaldsson A, Ostenfeld E, Roijer A, et al. Cardiac resynchronization therapy guided by echocardiography, MRI, and CT imaging: a randomized controlled study. JACC Clin Electrophysiol. 2020;6(10):1300-9.

8. Carlsson M, Ugander M, Heiberg $\mathrm{E}$, Arheden $\mathrm{H}$. The quantitative relationship between longitudinal and radial function in left, right, and total heart pumping in humans. Am J Physiol Heart Circ Physiol. 2007;293(1):H636-44

9. Carlsson M, Ugander M, Mosén H, Buhre T, Arheden H. Atrioventricular plane displacement is the major contributor to left ventricular pumping in healthy adults, athletes, and patients with dilated cardiomyopathy. Am J Physiol Heart Circ Physiol. 2007;292(3):H1452-9.

10. Stephensen SS, Ostenfeld E, Steding-Ehrenborg K, Thilén U, Heiberg E, Arheden $\mathrm{H}$, et al. Alterations in ventricular pumping in patients with atrial septal defect at rest, during dobutamine stress and after defect closure. Clin Physiol Funct Imaging. 2018;38(5):830-9.

11. Russell K, Eriksen M, Aaberge L, Wilhelmsen N, Skulstad H, Remme EW, et al. A novel clinical method for quantification of regional left ventricular pressure-strain loop area: a non-invasive index of myocardial work. Eur Heart J. 2012;33(6):724-33.

12. Rangarajan V, Chacko SJ, Romano S, Jue J, Jariwala N, Chung J, et al. Left ventricular long axis function assessed during cine-cardiovascular magnetic resonance is an independent predictor of adverse cardiac events. J Cardiovasc Magn Reson. 2016;18(1):35.

13. Ghani A, Delnoy PPHM, Ottervanger JP, Misier ARR, Smit JJJ, Adiyaman A, et al. Association of apical rocking with long-term major adverse cardiac events in patients undergoing cardiac resynchronization therapy. Eur Heart J Cardiovasc Imaging. 2016;17(2):146-53.

14. Khidir MJH, Abou R, Yilmaz D, Ajmone Marsan N, Delgado V, Bax JJ. Prognostic value of global longitudinal strain in heart failure patients treated with cardiac resynchronization therapy. Hear Rhythm. 2018;15(10):1533-9.

15. Borgquist R, Carlsson M, Markstad H, Ostenfeld E, Werther-Evaldsson A, Roijer A, et al. Cardiac resynchronization therapy guided by echocardiography, MRI and CT imaging - a randomized controlled study. JACC Clin Electrophysiol. 2020;6:1300-9.

16. Felker GM, Shaw LK, O'Connor CM. A standardized definition of ischemic cardiomyopathy for use in clinical research. J Am Coll Cardiol. 2002;39(2):210-8.

17. Heiberg E, Sjögren J, Ugander M, Carlsson M, Engblom H, Arheden H. Design and validation of segment — freely available software for cardiovascular image analysis. BMC Med Imaging. 2010;10(1):1.

18. Schulz-Menger J, Bluemke DA, Bremerich J, Flamm SD, Fogel MA, Friedrich $M G$, et al. Standardized image interpretation and post processing in cardiovascular magnetic resonance: Society for Cardiovascular Magnetic Resonance (SCMR) Board of Trustees Task Force on standardized post processing. J Cardiovasc Magn Reson. 2013;15(1):35.

19. Seemann F, Pahlm U, Steding-Ehrenborg K, Ostenfeld E, Erlinge D, Dubois-Rande $J$, et al. Time-resolved tracking of the atrioventricular plane displacement in Cardiovascular Magnetic Resonance (CMR) images. BMC Med Imaging. 2017;17(19):1-16.

20. Stephensen S, Steding-Ehrenborg K, Munkhammar P, Heiberg E, Arheden $\mathrm{H}$, Carlsson M. The relationship between longitudinal, lateral, and septal contribution to stroke volume in patients with pulmonary regurgitation and healthy volunteers. Am J Physiol Heart Circ Physiol. 2014;306(6):H895-903.

21. Lang RM, Badano LP, Mor-Avi V, Afilalo J, Armstrong A, Ernande L, et al. Recommendations for cardiac chamber quantification by echocardiography in adults: an update from the American society of echocardiography and the European association of cardiovascular imaging. J Am Soc Echocardiogr. 2015;28(1):233-71.

22. Stellbrink C, Breithardt O-A, Franke A, Sack S, Bakker P, Kramer A, et al. Impact of cardiac resynchronization therapy using hemodynamically optimized pacing on left ventricular remodeling in patients with congestive heart failure and ventricular conduction disturbances. J Am Coll Cardiol. 2001;38(7):1957-65.

23. Ypenburg C, van Bommel RJ, Borleffs CJW, Bleeker GB, Boersma E, Schalij $\mathrm{MJ}$, et al. Long-term prognosis after cardiac resynchronization therapy is related to the extent of left ventricular reverse remodeling at midterm follow-up. J Am Coll Cardiol. 2009;53(6):483-90. 
24. Revah G, Wu V, Huntjens PR, Piekarski E, Chyou JY, Axel L. Cardiovascular magnetic resonance features of mechanical dyssynchrony in patients with left bundle branch block. Int J Cardiovasc Imaging. 2016;32(9):1427-38.

25. Bursac Z, Gauss CH, Williams DK, Hosmer DW. Purposeful selection of variables in logistic regression. Source Code Biol Med. 2008;3:1-8.

26. Dupont WD, Plummer WD. Power and sample size calculations. A review and computer program. Control Clin Trials. 1990;11(2):116-28.

27. Zweerink A, van Everdingen WM, Nijveldt R, Salden OAE, Meine M, Maass $\mathrm{AH}$, et al. Strain imaging to predict response to cardiac resynchronization therapy: a systematic comparison of strain parameters using multiple imaging techniques. ESC Heart Fail. 2018;5:1130-40.

28. Rickard J, Michtalik H, Sharma R, Berger Z, lyoha E, Green AR, et al. Predictors of response to cardiac resynchronization therapy: a systematic review. Int J Cardiol. 2016;225:345-52.

29. Stankovic I, Prinz C, Ciarka A, Daraban AM, Kotrc M, Aarones M, et al. Relationship of visually assessed apical rocking and septal flash to response and long-term survival following cardiac resynchronization therapy (PREDICT-CRT). Eur Heart J Cardiovasc Imaging. 2016;17(3):262-9.

30. Lumens J, Tayal B, Walmsley J, Delgado-Montero A, Huntjens PR, Schwartzman D, et al. Differentiating electromechanical from nonelectrical substrates of mechanical discoordination to identify responders to cardiac resynchronization therapy. Circ Cardiovasc Imaging. 2015;8(9):1-12.

31. Ellims AH, Pfluger H, Elsik M, Butler MJ, Hare JL, Taylor AJ. Utility of cardiac magnetic resonance imaging, echocardiography and electrocardiography for the prediction of clinical response and long-term survival following cardiac resynchronisation therapy. Int J Cardiovasc Imaging. 2013;29(6):1303-11.

32. Galli E, Leclercq C, Fournet M, Hubert A, Bernard A, Smiseth OA, et al. Value of myocardial work estimation in the prediction of response to cardiac resynchronization therapy. J Am Soc Echocardiogr. 2018:31(2):220-30.

33. Delgado V, Van Bommel RJ, Bertini M, Borleffs CJW, Marsan NA, Ng ACT, et al. Relative merits of left ventricular dyssynchrony, left ventricular lead position, and myocardial scar to predict long-term survival of ischemic heart failure patients undergoing cardiac resynchronization therapy. Circulation. 2011;123(1):70-8.

34. Harb SC, Toro S, Bullen JA, Obuchowski NA, Xu B, Trulock KM, et al. Scar burden is an independent and incremental predictor of cardiac resynchronisation therapy response. Open Heart. 2019;6(2):11-4.

35. Sillanmäki S, Lipponen JA, Tarvainen MP, Laitinen T, Hedman M, Hedman A, et al. Relationships between electrical and mechanical dyssynchrony in patients with left bundle branch block and healthy controls. J Nucl Cardiol. 2018;38:1-12.

36. Brainin P, Hoffmann S, Fritz-Hansen T, Olsen FJ, Jensen JS, BieringSørensen T. Usefulness of postsystolic shortening to diagnose coronary artery disease and predict future cardiovascular events in stable angina pectoris. J Am Soc Echocardiogr. 2018;31(8):870-9.
37. Taylor AJ, Elsik M, Broughton A, Cherayath J, Leet A, Wong C, et al. Combined dyssynchrony and scar imaging with cardiac magnetic resonance imaging predicts clinical response and long-term prognosis following cardiac resynchronization therapy. Europace. 2010;12(5):708-13.

38. Auricchio A, Prinzen FW. Non-responders to cardiac resynchronization therapy. Circ J. 2011;75(3):521-7.

39. Van'T Sant J, Mast TP, Bos MM, Ter Horst IA, Van Everdingen WM, Meine M, et al. Echo response and clinical outcome in CRT patients. Neth Hear J. 2016:24(1):47-55.

40. Abraham WT, Young JB, León AR, Adler S, Bank AJ, Hall SA, et al. Effects of cardiac resynchronization on disease progression in patients with left ventricular systolic dysfunction, an indication for an implantable cardioverter-defibrillator, and mildly symptomatic chronic heart failure. Circulation. 2004;110(18):2864-8.

41. Maass AH, Vernooy K, Wijers SC, Van 'T Sant J, Cramer MJ, Meine M, et al. Refining success of cardiac resynchronization therapy using a simple score predicting the amount of reverse ventricular remodelling: results from the markers and response to CRT (MARC) study. Europace. 2018;20(2):e1-10.

42. Packer M. Proposal for a new clinical end point to evaluate the efficacy of drugs and devices in the treatment of chronic heart failure. J Card Fail. 2001;7(2):176-82.

43. Aalen JM, Donal E, Larsen CK, Duchenne J, Lederlin M, Cvijic M, et al. Imaging predictors of response to cardiac resynchronization therapy: left ventricular work asymmetry by echocardiography and septal viability by cardiac magnetic resonance. Eur Heart J. 2020;41:3813-23.

44. Abraham WT, Fisher WG, Smith AL, Delurgio DB, Leon AR, Loh E, et al. Cardiac resynchronization in chronic heart failure. N Engl J Med. 2002;346(24):1845-53.

45. Puntmann VO, Carr-White G, Jabbour A, Yu CY, Gebker R, Kelle S, et al. T1-mapping and outcome in nonischemic cardiomyopathy all-cause mortality and heart failure. JACC Cardiovasc Imaging. 2016;9(1):40-50.

46. Vita T, Gräni C, Abbasi SA, Neilan TG, Rowin E, Kaneko K, et al. Comparing CMR mapping methods and myocardial patterns toward heart failure outcomes in nonischemic dilated cardiomyopathy. JACC Cardiovasc Imaging. 2019;12(8P2):1659-69.

47. Chen Z, Sohal M, Sammut E, Child N, Jackson T, Claridge S, et al. Focal but not diffuse myocardial fibrosis burden quantification using cardiac magnetic resonance imaging predicts left ventricular reverse modeling following cardiac resynchronization therapy. J Cardiovasc Electrophysiol. 2016;27(2):203-9.

\section{Publisher's Note}

Springer Nature remains neutral with regard to jurisdictional claims in published maps and institutional affiliations.
Ready to submit your research? Choose BMC and benefit from:

- fast, convenient online submission

- thorough peer review by experienced researchers in your field

- rapid publication on acceptance

- support for research data, including large and complex data types

- gold Open Access which fosters wider collaboration and increased citations

- maximum visibility for your research: over $100 \mathrm{M}$ website views per year

At BMC, research is always in progress.

Learn more biomedcentral.com/submissions 